$$
\sum_{k=1}^{\infty} a_{k} \xi_{k}^{2} \sum_{k=1}^{\infty} \frac{1}{a_{k}} \xi_{k}^{2} \leqq \frac{(M+m)^{2}}{4 m M}\left[\sum_{k=1}^{\infty} \xi_{k}^{2}\right]^{2}
$$

for all $x \in l_{2}$. This is inequality (2).

\title{
REFERENCES
}

1. L. V. Kantorovich, Functional analysis and applied mathematics, Uspehi Mat. Nauk, vol. 3 (1948) pp. 89-185. Translated from the Russian by Curtis D. Benster, National Bureau of Standards, Report No. 1509, March 7, 1952.

2. G. P6lya and G. Szegö, Aufgaben und Lehrsätze aus der Analysis, Springer, Berlin, 2d ed., 1954, p. 57.

3. F. Riesz and B. Sz.-Nagy, Lectures on functional analysis, New York, F. Ungar Publishing Company, 1955.

Mathematisches Institut, Universität Zürich, Switzerland and National Bureau of Standards

\section{ON A PROBLEM OF LOHWATER}

\section{G. PIRANIAN}

Bagemihl [1] has shown that, for each complex-valued function $f$ defined in the unit disk $D$, there exist at most countably many points $e^{i \theta}$ that are endpoints of two Jordan $\operatorname{arcs} \gamma_{1}(\theta)$ and $\gamma_{2}(\theta)$ such that the corresponding cluster sets $C\left(f, e^{i \theta} ; \gamma_{1}(\theta)\right)$ and $C\left(f, e^{i \theta} ; \gamma_{2}(\theta)\right)$ are disjoint. Lohwater $[2$, p. 173] has recently asked whether there exists a function $f$ for which uncountably many points $e^{i \theta}$ are endpoints of three Jordan $\operatorname{arcs} \gamma_{1}(\theta), \gamma_{2}(\theta), \gamma_{3}(\theta)$ such that the intersection

$$
C\left(f, e^{i \theta} ; \gamma_{1}(\theta)\right) \cap C\left(f, e^{i \theta} ; \gamma_{2}(\theta)\right) \cap C\left(f, e^{i \theta} ; \gamma_{3}(\theta)\right)
$$

is empty. The property of points just described will be called the three-path property.

THEOREM. There exists a complex-valued function, continuous in the unit disk, for which each point $e^{i \theta}$ has the three-path property.

My proof is based on a slight modification of a technique recently used in connection with a problem on ambiguous points of a function defined in the unit sphere [3]. Let $T_{1}, T_{2}, T_{3}$ be three trees in $D$, with the property that each point $e^{i \theta}$ can be approached along three Jordan arcs which lie on $T_{1}, T_{2}, T_{3}$, respectively, except for their common endpoint $e^{i \theta}$. Let $T_{1}, T_{2}, T_{3}$ have the further property that no point

Received by the editors October 13, 1958. 
lies on all three of the trees, and that the set of points lying on more than one of the trees and in the disk $|z|<r$ is finite, for $r<1$.

Let $\omega=e^{2 i \pi / 3}$, and let $f(z)=\omega^{h+k}$ at all points of intersection of $T_{h}$ and $T_{k}(h, k=1,2,3 ; h \neq k)$. Clearly, $f$ can be defined on the three trees in such a way that it is continuous on each of them, and so that $f(z)=e^{i \phi(z)}$ with

$$
\begin{aligned}
& 0 \leqq \phi(z) \leqq 2 \pi / 3 \quad \text { for } z \text { on } T_{1}, \\
& 4 \pi / 3 \leqq \phi(z) \leqq 2 \pi \quad \text { for } z \text { on } T_{2} \text {, } \\
& 2 \pi / 3 \leqq \phi(z) \leqq 4 \pi / 3 \quad \text { for } z \text { on } T_{3} \text {. }
\end{aligned}
$$

The continuous extension of $f(z)$ into the remainder of the open unit disk presents no difficulty, and the proof is complete.

Added in Proof. A forthcoming paper by F. Bagemihl, G. Piranian and G. S. Young will exhibit a bounded holomorphic function for which uncountably many points $e^{i \theta}$ have the three-arc property.

\section{REFERENCES}

1. F. Bagemihl, Curvilinear cluster sets of arbitrary functions, Proc. Nat. Acad. Sci. U.S.A. vol. 41 (1955) pp. 379-382.

2. A. J. Lohwater, The cluster sets of meromorphic functions, Thirteenth Congress Math. Scand. (1957) pp. 171-177.

3. G. Piranian, Ambiguous points of a function continuous inside a sphere, Michigan Math. J. vol. 4 (1957) pp. 151-152.

The University of Michigan 